\title{
L'apprentissage par problèmes et par projets pour le développement des compétences professionnelles de l'ingénieur : l'exemple des programmes de gé- nie électrique et de génie informatique de l'Université de Sherbrooke
}

\author{
Noël Boutin ${ }^{1}$, Daniel Dalle ${ }^{1}$, Gérard Lachiver ${ }^{1}$ et Philippe Mabilleau \\ Université de Sherbrooke, Faculté de génie, 2500 Boul. de L’Université, Sherbrooke, Québec, Canada, J1K 2R1
}

Noel.Boutin@USherbrooke.ca

\begin{abstract}
RÉSUMÉ :
Cet article présente les rénovations apportées aux programmes de baccalauréat en génie électrique et en génie informatique de l'Université de Sherbrooke dans le but de développer les compétences professionnelles de l'ingénieur. Cinq innovations pédagogiques sont mises de l'avant : adoption d'une approche par compétences, caractérisation de chaque session d'étude par un thème, prise en charge de chaque session par une équipe de formateurs, apprentissage par problèmes et par projets sur tout le curriculum des deux programmes, ajout à la méthode APP classique de périodes collaboratives de formation à la pratique procédurale et de formation à la pratique en laboratoire.
\end{abstract}

Mots clés: Rénovations des curricula, génie électrique, génie informatique, compétences professionnelles de l'ingénieur, approche par compétences, équipes professorales, apprentissage par problèmes et par projets, période de formation à la pratique procédurale, période de formation à la pratique en laboratoire.

\section{INTRODUCTION}

Les exigences de la profession d'ingénieur se sont fortement modifiées au cours de la dernière décennie sous la forte pression d'un environnement économique en mutation et de problèmes de plus en plus complexes à résoudre. Selon Le Boterf, « un professionnel est celui qui sait gérer un ensemble de situations professionnelles dont la plupart se caractérisent par leur complexité et leur caractère inédit $\gg[1]$.

Les ingénieurs doivent dorénavant non seulement démontrer de solides compétences scientifiques mais également des compétences en communication, en travail en équipe, en gestion de projets, en créativité, tout en exerçant leur profession de façon socialement responsable, avec un sens accru de l'éthique et dans un souci de respect de l'environnement [2-3].

Pour répondre à ces nouvelles exigences, le Département de génie électrique et de génie informatique de l'Université de Sherbrooke a complètement rénové ses deux programmes de formation des ingénieurs. Cinq innovations pédagogiques ont été mises de l'avant: adoption d'une approche par compétences, caractérisation de chaque session d'étude par un thème, prise en charge de chaque session par une équipe de formateurs, apprentissage par problèmes et par projets sur l'ensemble des deux curricula, ajout de périodes collaboratives de formation à la pratique procédurale et de formation à la pratique en laboratoire. Les lignes qui suivent présentent chacune de ces cinq innovations.

1 Noël Boutin, Daniel Dalle et Gérard Lachiver sont membres du Centre d'études et de recherche en enseignement supérieur (CERES)

\section{ADOPTION D'UNE APPROCHE PAR COMPETENCES}

Il a été décidé de rénover les deux programmes suivant une approche par compétences. Plusieurs définitions de ce qu'est une compétence furent proposées dans la littérature scientifique au fil des années. Une des plus récentes est celle de Tardif [4] : «Une compétence est un savoir-agir complexe qui prend appui sur la mobilisation efficace d'une variété de ressources internes et externes à l'intérieur d'une famille de situations ».

Quatre catégories de compétences attendues des finissants de chacun des deux programmes furent retenues :

- Compétences scientifiques et techniques propres à chaque programme

- Compétences en conception communes aux deux programmes

- Compétences interpersonnelles communes aux deux programmes

- Compétences intra personnelles communes aux deux programmes

Une fois les compétences établies, celles-ci furent projetées sur l'ensemble de chaque programme en respectant la structure en place, soit une formation en alternance de 120 crédits comportant 8 sessions d'études ( $\mathrm{S} 1$ à $\mathrm{S} 8$ ) entre lesquelles s'insèrent 5 stages ( $\mathrm{T} 1$ à $\mathrm{T} 5$ ) de 4 mois à partir de la deuxième session, comme l'illustre la figure 1. Les six premières sessions construisent l'essentiel des compétences de chaque programme et amènent les étudiants à apprendre de façon autonome. Lors des dernières sessions, les compétences en conception sont complétées grâce à un projet de grande 
envergure et les étudiants ont la possibilité de choisir des modules de spécialisation. Les stages en industrie complètent la construction des compétences en favorisant l'intégration des connaissances.

\begin{tabular}{|c|c|c|c|c|c|c|c|c|c|}
\hline Anné & & Année & e 2 & & Innée & & & nnée 4 & \\
\hline \begin{tabular}{l|l|} 
Aut & Hiv \\
\end{tabular} & Été & \begin{tabular}{|l|l|} 
Aut & Hiv \\
\end{tabular} & Été & Aut & $\mathrm{Hiv}$ & Été & Aut & $5 n$ & Aut \\
\hline \begin{tabular}{l|l|} 
S1 & S2 \\
\end{tabular} & $\mathrm{T} 1$ & \begin{tabular}{|l|l|} 
S3 & T2 \\
\end{tabular} & S4 & S5 & \begin{tabular}{l|l} 
T3 & $S$ \\
\end{tabular} & S6 & $\mathrm{T} 4$ & \begin{tabular}{|l|l|}
$S 7$ & $T$ \\
\end{tabular} & S8 \\
\hline
\end{tabular}

\section{Figure 1: Agencement typique des sessions d'études et des stages de travail}

\section{3}

\section{THEME POUR CHAQUE SESSION}

Dans une approche par compétences, les apprentissages ne sont pas cloisonnés mais plutôt intégrés afin de construire des compétences spécifiques. Il a donc fallu repenser le cadre de chaque session d'études existante composée typiquement de 5 activités pédagogiques de 3 crédits, le plus souvent sans aucun lien les unes avec les autres. Une solution originale fut retenue. Chaque session d'étude est caractérisée par un thème différent qui définit le domaine d'application des compétences développées et fournit le cadre pratique des situations d'apprentissage faisant référence à la pratique professionnelle.

Le tableau 1 présente les thèmes retenus pour les 6 premières sessions de chaque programme, les deux dernières sessions étant consacrées à un domaine de spécialisation au choix de l'étudiant et à un projet majeur de conception. La première session est commune aux 2 programmes afin de permettre aux étudiants de prendre contact avec les deux champs de pratique professionnelle et de faire un choix plus éclairé par la suite.

\begin{tabular}{|l|l|}
\cline { 2 - 2 } \multicolumn{1}{c|}{} & \multicolumn{1}{c|}{ Génie électrique } \\
\hline Session 1 & Introduction au génie élec. et génie info. \\
\hline Session 2 & Signaux et circuits \\
\hline Session 3 & Systèmes électriques et électroniques \\
\hline Session 4 & Asservissements \\
\hline Session 5 & Systèmes et circuits numériques \\
\hline Session 6 & Systèmes de télécommunications \\
\hline
\end{tabular}

\begin{tabular}{|l|l|}
\cline { 2 - 2 } \multicolumn{1}{c|}{} & \multicolumn{1}{c|}{ Génie informatique } \\
\hline Session 1 & Introduction au génie élec. et génie info. \\
\hline Session 2 & Systèmes informatiques \\
\hline Session 3 & Architecture des systèmes \\
\hline Session 4 & Ingénierie des systèmes \\
\hline Session 5 & Systèmes embarqués \\
\hline Session 6 & Systèmes téléinformatiques \\
\hline
\end{tabular}

\section{Tableau 1 : Thèmes des sessions d'études de chaque programme}

La conception et la prestation de chaque session sont prises en charge par une équipe de formateurs constituée de professeurs du département et, au besoin, complétée par des chargés de cours ou des spécialistes de domaines autres que le génie électrique et informatique. La composition de l'équipe professorale dépend du thème de la session et des compétences qu'elle vise à développer.

Durant le déroulement d'une session, chaque équipe de formateurs se réunit une fois par semaine pour faire le point et le suivi. Un des professeurs membres de l'équipe assure la coordination de l'équipe, gère le site web de sa session et recueille périodiquement les commentaires des étudiants.

\section{L'APPRENTISSAGE PAR PROBLEMES ET PAR PROJETS}

Les méthodes pédagogiques traditionnelles, basées principalement sur la leçon magistrale, s'avèrent inadéquates pour la construction des compétences. En effet, comme le souligne Mary E. Huba et Jann E. Freed, [5] : " Those among us who espouse traditional approaches to teaching feel responsible for imparting to students the most current information in our fields. We typically use the lecture method of instruction that require the professor to seek out new information, integrate it with existing knowledge, organize it for presentation to students, and explain it orally to the class. ... From a constructivist perspective, the individuals learning the most in this classroom are the professors. They have reserve for themself the very conditions that promote learning: actively seeking new information, integrating it what is known, organizing it in a meaningful way, and having the chance to explain it to others. »)

Il a donc fallu chercher d'autres approches pédagogiques que la leçon magistrale. Selon R. L. Côté [6] : " ... les grands maîtres $n$ 'ont pas enseigné, dans le sens de transmettre des connaissances, mais ont été des personnes qui ont su susciter des questionnements chez leurs disciples et les ont aidés à découvrir la connaissance et ainsi à construire les différentes formes de savoirs. Leur préoccupation principale a été de redonner à l'apprenant le pouvoir sur son processus d'apprentissage. »

Une place importante doit donc être accordée à la métacognition qui, selon L. Lafortune et al [7], «... consiste à simultanément agir et prendre conscience de sa propre démarche pour s'ouvrir et s'enrichir de celle des autres. Développer cette distanciation de sa propre action se fait en déterminant des tâches qui 
rendent l'élève actif et attentif à sa propre démarche d'apprentissage, qui le sensibilisent à ses forces et lui font penser aux actions qu'il doit entreprendre pour compenser ses faiblesses. La métacognition est alors centrée sur le regard que la personne pose sur sa démarche mentale. Elle ne relève pas du domaine exclusif d'une discipline et vise le transfert progressif à l'élève de la responsabilité de l'apprentissage. ».

L'apprentissage par problèmes et l'apprentissage par projets sont des approches pédagogiques favorisant la métacognition. Les étudiants sont actifs et responsables de leurs propres apprentissages. La construction des connaissances et des compétences est non seulement personnelle, mais possède une dimension sociale. Les étudiants travaillent en groupes collaboratifs plutôt qu'individuellement, ils sont amenés à échanger sur leurs stratégies d'apprentissages, à collaborer pour diverses activités de formation, et à coopérer en équipe pour réaliser des projets. Selon Jacques Tardif [8], « La résolution de problèmes devrait constituer la pierre angulaire du curriculum scolaire». D.R. Woods, un des pionniers, au Canada, à utiliser l'apprentissage par problèmes dans des cours de génie chimique affirme que [9]: «Problem-based learning $(P B L)$ is one of the major success stories in education since the 1970s. You are fortunate, indeed, if you are learning via the PBL mode. »

Le projet consistait à mettre en œuvre, non pas sur un seul cours ou sur quelques cours, mais bien sur tout le curriculum, l'apprentissage par problèmes (APP) jumelé avec l'apprentissage par projet. En absence de références pratiques sur des réalisations d'une telle envergure dans des programmes de formation en génie, il a donc fallu innover. Ceci constitue la quatrième innovation que nous avons convenu d'appeler l'Apprentissage par Problèmes et par Projets en Ingénierie (APPI). Comme pour les programmes de formation universitaires conventionnels, l'étudiant s'inscrit à des activités pédagogiques (AP) décrites à l'annuaire [10] par des éléments de compétences et des contenus faisant l'objet des apprentissages de chaque session. Toutefois, contrairement aux programmes de formation conventionnels, il n'y a pas un cours associé à chaque AP, mais plutôt une séquence d'unités d'apprentissage par problèmes (APP) collaboratif, chacune d'une durée typique de deux semaines, qui intègrent les apprentissages décrits à l'annuaire. Chaque unité d'APP incorpore, dans un tout cohérent, des fragments d'AP, contribue au développement des compétences de la session et développe les connaissances qui sont intégrées au fur et à mesure dans un projet parallèle d'équipe s'échelonnant sur toute la session. Ce projet d'intégration s'inscrit dans le thème de la session tel que défini dans la structure du programme [14]. La figure 2 illustre la structure générale d'une session du programme de formation d'une durée de 15 semaines.

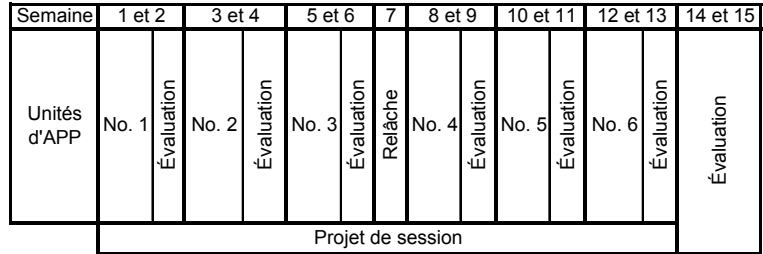

Figure 2: Structure typique d'une session d'études

Le projet s'écarte sur plusieurs points du modèle traditionnel de l'APP tel que mis en œuvre dans plusieurs programmes de formation médicale à travers le monde pour mieux l'adapter au programme de formation en génie.

D'abord, chaque unité d'APP dure deux semaines plutôt que quelques jours. Ceci permet de moins morceler les contenus, confrontant ainsi les étudiants à une problématique suffisamment riche, englobant plusieurs facettes. Chaque équipe professorale de session a la responsabilité d'écrire les problématiques. Il s'agit d'une responsabilité partagée au sens où une problématique écrite par un professeur est d'abord partagée avec les autres membres de l'équipe professorale de session pour commentaires et bonification avant d'être soumise aux étudiants. Typiquement, le texte de chaque problématique s'étend sur deux pages et demande de la part de l'étudiant, pour sa résolution, une acquisition de nouvelles connaissances contenues dans environ une centaine de pages de livres par semaine [15]. Ce rythme d'acquisition des connaissances est comparable à ce qu'il était avec l'approche conventionnelle ; pour la résolution d'une problématique il correspond environ au volume de connaissances associé à la moitié d'un cours de 45 heures. Il est important de souligner que c'est le développement de compétences qui est attendu de la part de l'étudiant et non pas l'accumulation brute de connaissances.

\section{PERIODES COLLABORATIVES DE FORMATION A LA PRATIQUE PROCE- DURALE ET EN LABORATOIRE}

Comme en génie, l'acquisition uniquement de connaissances déclaratives ne suffit pas, chaque unité d'APP a été bonifiée de périodes collaboratives de formation à la pratique procédurale et de formation à la pratique en laboratoire. La figure 3 illustre l'horaire type d'une unité de deux semaines. Les périodes de formation à la pratique procédurale interviennent après des périodes importantes d'étude individuelle des étudiants. Lors de ces pratiques procédurales, un groupe formé typiquement de 48 étudiants regroupés en cellules de huit autour d'une table, collabore durant 3 heures à la résolution de problèmes soumis par un professeur. Pour chaque problème, après un certain temps laissé pour sa résolution et un partage au sein du groupe collaboratif, un étudiant est invité à présenter 
au tableau sa solution. Le tout se déroule dans un climat de collaboration, sans aucune compétitivité, où le droit à l'erreur fait partie intégrante du processus de formation car, comme le mentionne Donald A. Schön [11]: "Admettre qu'on s'est trompé, c'est ouvrir la porte de l'incertitude, mais c'est aussi ouvrir celle de la découverte plutôt que celle de l'auto-défense ». De plus, comme le soulignent Olivier Clouzot et Annie Bloch [12]: "Personne ne peut faire un apprentissage à la place d'un autre, mais nous apprenons souvent avec l'autre, grâce à lui, à cause de lui ». Nous reconnaissons que la collaboration joue un rôle important dans la formation car, tout comme le pense R. L. Côté [13] : «... le fait d'essayer de décrire à une autre personne notre compréhension d'une expérience ou d'une réalité permet souvent de réaliser les limites de notre représentation $\gg$. Durant ces périodes, le professeur valide les solutions proposées par les étudiants et, au besoin, apporte un complément d'information. Dans les sessions où le nombre d'étudiants dépasse 48, deux professeurs se partagent la tâche d'animer les périodes parallèles de formation à la pratique procédurale dans des locaux différents. Tous deux conviennent ensemble des problèmes soumis aux étudiants et utilisent le même guide du formateur. Une fois la rencontre avec les étudiants terminée, ils font ensemble un bilan de leur perception respective de la progression des étudiants dans leur apprentissage.

\begin{tabular}{|c|c|c|c|c|}
\hline Lundi & Mardi & Mercredi & Jeudi & Vendredi \\
\hline \multirow{3}{*}{$\begin{array}{c}\text { Tutorat } \\
\text { Première } \\
\text { rencontre }\end{array}$} & \multirow{3}{*}{ Étude } & & \multirow{3}{*}{ Projet } & Rétroaction \\
\hline & & $\begin{array}{c}\text { Formation } \\
\text { Pratique } \\
\text { procédurale }\end{array}$ & & \multirow[t]{2}{*}{ Étude } \\
\hline & & Séminaire & & \\
\hline \multirow[b]{2}{*}{ Étude } & \multirow[b]{2}{*}{ Étude } & \multirow{2}{*}{$\begin{array}{l}\text { Formation } \\
\text { Pratique en } \\
\text { laboratoire }\end{array}$} & \multirow[b]{2}{*}{ Étude } & $\begin{array}{c}\text { Travail } \\
\text { collaboratif }\end{array}$ \\
\hline & & & & Étude \\
\hline
\end{tabular}

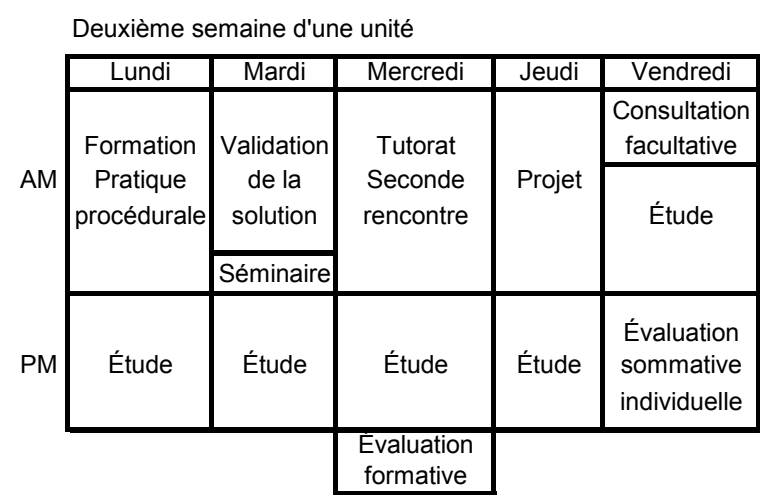

Figure 3 : Horaire type d'une unité d'APP

\section{7}

\section{CONCLUSION}

Les innovations pédagogiques présentées ici ont été mises en oeuvre au sein des programmes de baccalauréat en génie électrique et en génie informatique depuis septembre 2001. Deux cohortes d'étudiants ayant bénéficié de cette nouvelle formule pédagogique tout au long de leur cheminement ont diplômé en décembre 2005 et décembre 2006. L'accueil donné à ces innovations est très positif autant du point de vue des étudiants, auxquels des mécanismes de rétroaction sont offerts à de nombreuses occasions, que par le feedback indirect des employeurs qui ont observé une amélioration de l'autonomie professionnelle des stagiaires et des finissants. Ces innovations permettent une meilleure adéquation entre la formation donnée aux nouveaux ingénieurs et les exigences de la profession dans un secteur en évolution rapide où l'autonomie d'apprentissage et le travail en équipe sont des éléments clés. Les habilités sociales développées en situation de production et d'apprentissage chez les étudiants dans l'APPI, couplées à des compétences scientifiques et techniques solides, sont des atouts qui leur donnent un avantage déterminant pour entrer dans leur vie professionnelle.

\section{Bibliographie}

[1] G. Le Boterf, Développer la compétence des professionnels, 4ième édition, Éditions d'Organisation, 2002.

[2] Canadian Academy of Engineering, « Evolution of Engineering Education in Canada », 1999. http://www.acad-enggen.ca/publis/publi_an.html

[3] M. Besterfield-Sacre, L.J. Shuman, H. Wolfe, C.J. Atman, J. McGourty, R.L. Miller, B.M. Olds, G.M. Rogers, "Defining the Outcomes:A Framework for EC-2000», IEEE Trans. on Education, vol. 43, no.2, may 2000, p.100-110.

[4] J. Tardif, L'évaluation des compétences, Chenelière Éducation, 2006.

[5] Mary E. Huba et Jann E. Freed, « LearnerCentered Assessment on College Campuses. Shifting the focus from teaching to learning », Allyn and Bacon, 2000, Ma, p. 35

R. L. Côté, «Apprendre - Formation expérientielle stratégique », Presses de l’Université du Québec, Ste-Foy, 1998, p. 5.

[7] L. Lafortune et al, « Pour guider la métacognition », Presses de l'Université du Québec, Ste-Foy, 2000, p. 8. 
[8] J. Tardif, « Pour un enseignement stratégique - l'apport de la psychologie cognitive », Les Éditions Logiques, Montréal, 1992, p. 217.

[9] D. R. Woods, «Problem-based Learning : How to gain the most from PBL », D.R. Woods, Waterdown, ON. ,1994, p. ix.

[10] Université de Sherbrooke. Description des programmes de génie informatique et de génie électrique. 2006.

http://www.usherbrooke.ca/Programmes/bacc /gelectri.html;

http://www.usherbrooke.ca/Programmes/bacc /ginform.html.

[11] Donald A. Schön, «Le praticien réflexif », Les Éditions Logiques, 1994, Montréal, p. 66

[12] Olivier Clouzot et Annie Bloch, « Apprendre autrement », Les Éditions d'organisation, $\mathrm{Pa}$ ris, 1981, p. 101.

[13] R. L. Côté, «Apprendre - Formation expérientielle stratégique », Presses de l'Université du Québec, Ste-Foy, 1998, p. 47

[14] P. Mabilleau, « Le développement de compétences en conception de systèmes embarqués via l'apprentissage par problèmes et par projets », soumis au 6ième Colloque sur l'Enseignement des Technologies et des Sciences de l'Information et des Systèmes CETSIS, 2007

[15] Ahmed Khoumsi et Ruben Gonzalez-Rubio, «Applying a Competency- and ProblemBased Approach for Learning Compiler Design », Journal of STEM Education, Volume 7 Issue 1 \& 2 January-June 2006, p. 24-33. 